\title{
New Approach to Non-Invasive Assessment of Vascular Circulation Based on the Response to Transient Ischemia
}

\author{
Joanna Katarzynska $\mathbb{D}^{1}$, Jacek Zielinski $\mathbb{D}^{2}$, Andrzej Marcinek $\mathbb{D}^{1,3}$, Jerzy Gebicki $\mathbb{D}^{1,3}$ \\ 'Angionica Ltd., Lodz, Poland; ${ }^{2}$ Department of Athletics, Strength and Conditioning, Poznan University of Physical Education, Poznan, Poland; \\ ${ }^{3}$ Institute of Applied Radiation Chemistry, Lodz University of Technology, Lodz, Poland \\ Correspondence: Jerzy Gebicki; Andrzej Marcinek, Institute of Applied Radiation Chemistry, Lodz University of Technology, Lodz, 90-924, Poland, \\ Tel +48 42 63I 3I 7I; +48 42 63I 30 96, Email jerzy.gebicki@p.lodz.pl; andrzej.marcinek@p.lodz.pl
}

\section{Introduction}

The treatment of vascular diseases incurs tremendous cost to healthcare systems. Current approaches focus largely on managing symptoms. In the last decade, substantial evidence has linked vascular diseases with a dysfunctional response to hypoxia. A distinguishable reaction to hypoxia can be identified from macro- and microvessels. Based on this effect, we have developed a new, non-invasive diagnostic technique called Flow Mediated Skin Fluorescence (FMSF) to be used for assessment of vascular circulation and metabolic regulation. ${ }^{1}$ The FMSF technique has been successfully used for non-invasive diagnostics of many vascular diseases, including CVD, PAD, diabetes, and hypertension. ${ }^{2-4}$ The FMSF technique has also attracted a considerable interest among sports physiologists. ${ }^{5}$

One conclusion from our studies using the FMSF technique is that diagnostics of vascular circulation should focus not only on dysfunctional blood flow in major arteries, but also on microcirculatory responses to hypoxia. In this communication, we present two key parameters derived from FMSF measurements: Reactive Hyperemia Response (RHR) and Hypoxia Sensitivity $[\log (\mathrm{HS})]$. These parameters can be used for efficient characterization of vascular circulation based on the response to transient ischemia.

\section{Methods}

Measurements were performed using the AngioExpert, a device constructed by Angionica Ltd. The AngioExpert device uses the Flow Mediated Skin Fluorescence (FMSF) technique, which measures changes in the intensity of nicotinamide adenine dinucleotide (NADH) fluorescence from the skin on the forearm in response to blocking and releasing blood flow. The skin is the largest organ of the human body, and is characterized by specific metabolism. The epidermal layer of the skin is not directly vascularized, and oxygen and nutrients are transported from the dermis by diffusion. Therefore, epidermal cell metabolism can be considered as a unique and sensitive marker of early dysfunction in vascular circulation and metabolic regulation.

The AngioExpert assesses the patient's condition by analyzing changes in the NADH fluorescence signal emitted from epidermal cells in response to forced occlusion ischemia and subsequent hyperemia due to the removal of the occlusion. By interpreting the parameters and the shape of the obtained NADH fluorescence curve, disorders of the vascular circulation can be identified that may be indicative of chronic diseases. The AngioExpert measures the NADH fluorescence excited by ultraviolet (UV) radiation with a wavelength of $340 \mathrm{~nm}$ (UVB). As the maximum UVB penetration light of $340 \mathrm{~nm}$ is approximately $0.3-0.5 \mathrm{~mm}$, the results are determined by NADH fluorescence within the epidermis. The emitted wavelength of NADH fluorescence is $460 \mathrm{~nm}$ (blue light). The test is performed in a comfortable sitting position, after a minimum adaptation period of 5 minutes, in a quiet room with a controlled air temperature $\left(24 \pm 1{ }^{\circ} \mathrm{C}\right)$. The resting NADH fluorescence value emitted by the epidermal layer of the forearm is recorded 
for the first 3 minutes (180 s). The brachial artery is then occluded by inflating the cuff of the device to $60 \mathrm{mmHg}$ above the systolic pressure. The ischemic response is recorded over a period of 3 minutes $(180 \mathrm{~s})$. During this time, ischemic changes in the NADH fluorescence signal are recorded. Upon completion of the occlusion, the cuff pressure is released abruptly, restoring flow in the brachial artery and inducing a hyperemic response, for a minimum duration of 4 minutes (240 s).

RHR is a newly introduced parameter (defined in Figure 1), which characterizes endothelial function related predominantly to the production of nitric oxide (NO) in the vasculature due to reactive hyperemia. RHR is a unique parameter, based on the combined response from both the ischemic and hyperemic parts of the measured FMSF trace.

The HS parameter has been used previously in the analysis of FMSF traces and represents a direct measure of the intensity of microcirculatory oscillations related to myogenic oscillations with frequencies in the range of $0.052-0.15 \mathrm{~Hz}$, recorded during reperfusion. ${ }^{6,7}$ Myogenic microcirculatory oscillations are a very sensitive measure of the microcirculatory response to hypoxia, which can be monitored with high precision using the FMSF technique. As the values of the HS parameter can vary within a quite broad range it is more practical to use a normally distributed $\log (\mathrm{HS})$.

The study was conducted at the Medical University of Lodz, Poland, and Poznan University of Physical Education, Poland. It conformed to the principles outlined in the Declaration of Helsinki and the study protocol was approved by the University Bioethics Committee. All the subjects gave written informed consent prior to participation.

\section{Results and Discussion}

The results summarized in Figure 2 were collected for three studied groups: A - endurance athletes; B - healthy middleaged individuals; $\mathrm{C}$ - diabetes type 2 patients. The RHR parameter distinguishes these three groups with high statistical significance. Such observations indicate that the use of RHR parameter has adequate sensitivity to be used for characterization of vascular circulation. A similar conclusion can be formulated based on analysis of the $\log (\mathrm{HS})$ parameter, representing reaction of microcirculation to transient hypoxia. The parameters RHR and $\log (\mathrm{HS})$ describe quite distinctive properties of the vascular system, and both parameters should be used jointly for effective diagnostics. Some correlation exists between the $\log (\mathrm{HS})$ and RHR parameters for healthy individuals (groups A + B), as shown in

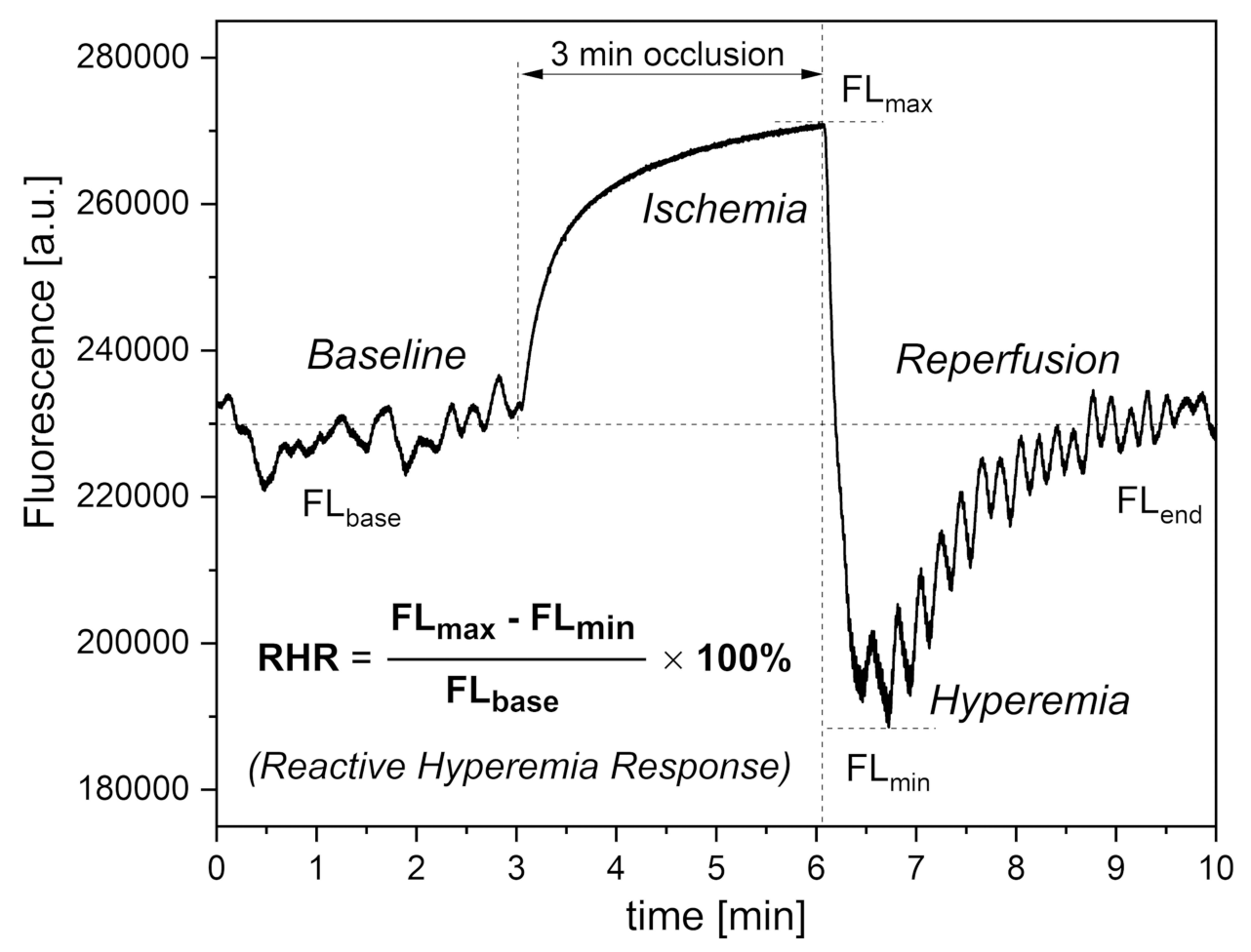

Figure I Definition of the RHR parameter. 
A

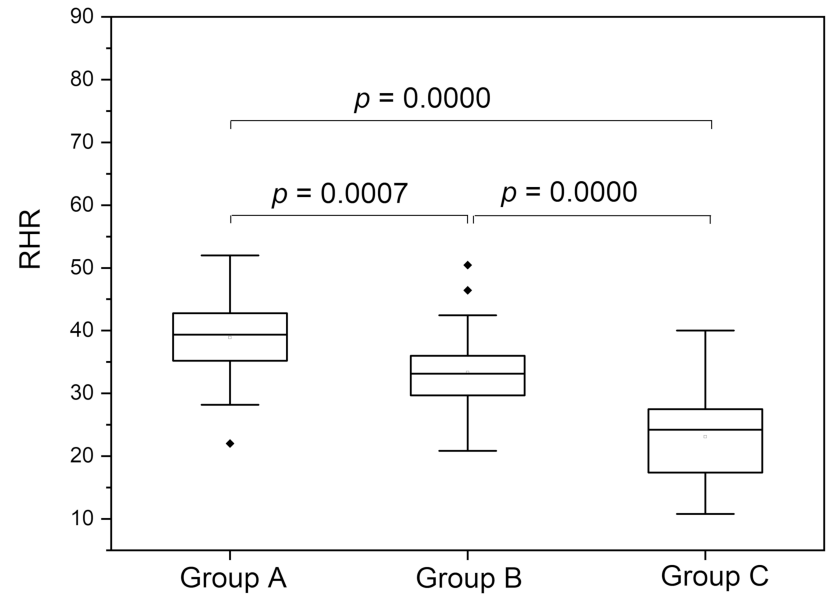

C

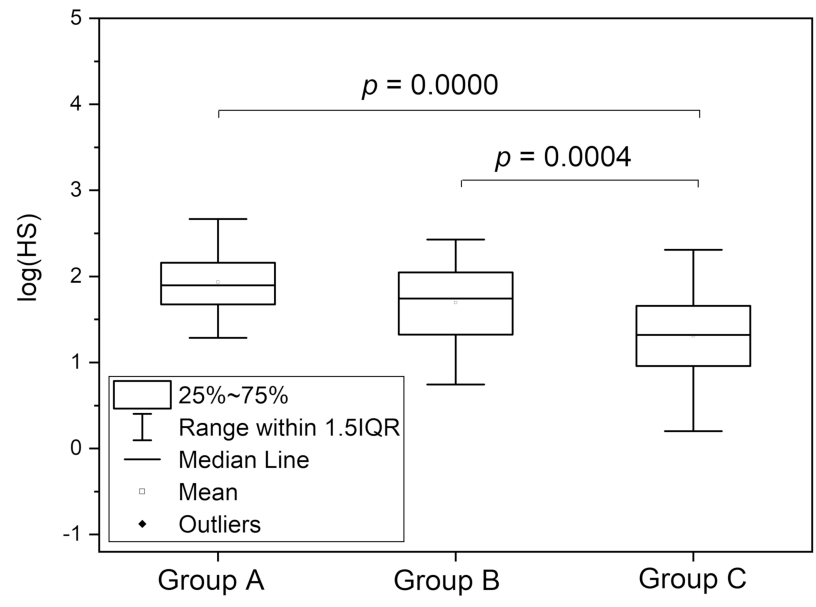

B

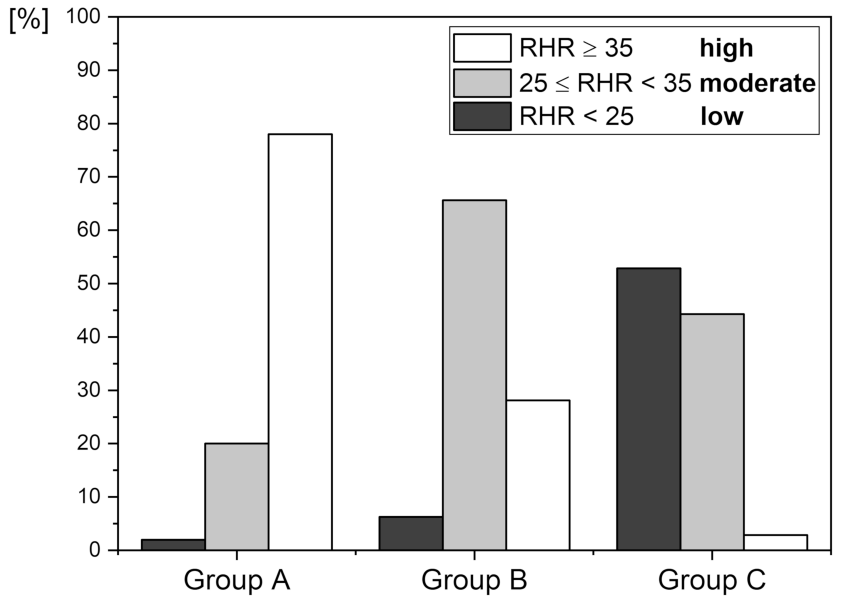

D

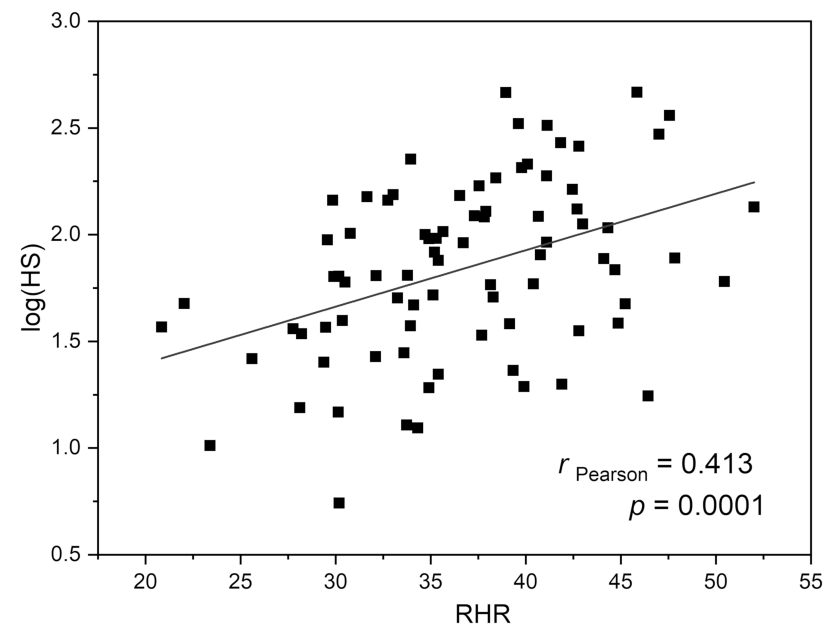

Figure 2 Assessment of RHR and $\log (\mathrm{HS})$ parameters in groups $\mathrm{A}, \mathrm{B}$, and C: A - highly trained endurance athletes (long-distance runners - 22, triathletes - I4, rowers - I4), $\mathrm{n}=50$ (33 m, $17 \mathrm{f}$ ), mean age 22.0 ( $16-35 \mathrm{y}$ ); $\mathrm{B}$ - healthy middle-aged individuals, $\mathrm{n}=32$ ( $19 \mathrm{~m}, \mathrm{I} \mathrm{f}$ ), mean age 38.2 ( $30-50 \mathrm{y}$ ); C - diabetes type 2 patients, $\mathrm{n}=70$ (40 m, 32 $\mathrm{f}$ ), mean age 63.1 (45-80 y.). Statistical analysis of differences between the parameters in the compared groups was based on the one-way ANOVA test with the Scheffe post hoc test. (A) Statistical differentiation of groups A, B, and C based on the RHR parameter. (B) Distribution of the RHR parameter. (C) Statistical differentiation of groups A, $B$, and $C$ based on the $\log (\mathrm{HS})$ parameter. (D) Correlation between the $\log (\mathrm{HS})$ and RHR parameters for groups $A+B$ (Pearson correlation).

Figure 2D. However, these parameters should be interpreted separately when analyzing the patients with various diseases and disorders of vascular origin. For example, very low values (below 1) for the $\log (\mathrm{HS})$ parameter can effectively predict a limited chance for healing in patients with diabetic foot ulcers, regardless of the measured value of the RHR parameter. ${ }^{4}$ Diagnostic use of the RHR parameter can be greater in CVD, where dysfunction in macrocirculation prevails.

Based on our experience using the FMSF diagnostic technique, we conclude that characterization of vascular circulation based on the RHR and $\log (\mathrm{HS})$ parameters can be effective across a broad segment of the population, from physically active healthy individuals to individuals suffering from serious health problems related to vascular dysfunctions. We believe that this simple two-parametric approach based on distinguishable macro- and microcirculatory responses to hypoxia will be recognized in the near future as a powerful diagnostic tool for characterization of vascular circulation.

\section{Funding}

This work was supported by the European Union from the resources of the European Regional Development Fund under the Smart Growth Operational Program, Grant No. POIR. 01.01.01-00-0540/15-00. 


\section{Disclosure}

JG and AM are inventors of the patents protecting the use of FMSF technology (EP2713860B1) issued to Angionica Ltd. Joanna Katarzynska, Andrzej Marcinek, and Jerzy Gebicki affiliated with Angionica Ltd. The authors report no other conflicts of interest in this work.

\section{References}

1. Katarzynska J, Lipinski Z, Cholewinski T, et al. Non-invasive evaluation of microcirculation and metabolic regulation using flow mediated skin fluorescence (FMSF): technical aspects and methodology. Rev Sci Instrum. 2019;90:104104. doi:10.1063/1.5092218

2. Katarzynska J, Borkowska A, Czajkowski P, et al. Flow Mediated Skin Fluorescence technique reveals remarkable effect of age on microcirculation and metabolic regulation in type 1 diabetes. Microvasc Res. 2019;124:19-24. doi:10.1016/j.mvr.2019.02.005

3. Katarzynska J, Borkowska A, Los A, et al. Flow-Mediated Skin Fluorescence (FMSF) technique for studying vascular complications in Type 2 diabetes. J Diabetes Sci Technol. 2020;14:693-694. doi:10.1177/1932296819895544

4. Los-Stegienta A, Katarzynska J, Borkowska A, et al. Differentiation of diabetic foot ulcers based on stimulation of myogenic oscillations by transient ischemia. Vasc Health Risk Manag. 2021;17:145-152. doi:10.2147/VHRM.S307366

5. Bugaj O, Zielinski J, Kusy K, et al. The effect of exercise on the skin content of the reduced form of NAD and its response to transient ischemia and reperfusion in highly trained athletes. Front Physiol. 2019;10:600. doi:10.3389/fphys.2019.00600

6. Gebicki J, Katarzynska J, Cholewinski T, et al. Flowmotion monitored by Flow Mediated Skin Fluorescence (FMSF): a tool for characterization of microcirculatory status. Front Physiol. 2020;11:702. doi:10.3389/fphys.2020.00702

7. Gebicki J, Marcinek A, Zielinski J. Assessment of microcirculatory status based on stimulation of myogenic oscillations by transient ischemia: from health to disease. Vasc Health Risk Manag. 2021;17:33-36. doi:10.2147/VHRM.S292087

\section{Publish your work in this journal}

Vascular Health and Risk Management is an international, peer-reviewed journal of therapeutics and risk management, focusing on concise rapid reporting of clinical studies on the processes involved in the maintenance of vascular health; the monitoring, prevention and treatment of vascular disease and its sequelae; and the involvement of metabolic disorders, particularly diabetes. This journal is indexed on PubMed Central and MedLine. The manuscript management system is completely online and includes a very quick and fair peer-review system, which is all easy to use. Visit http://www.dovepress.com/testimonials.php to read real quotes from published authors. 